\title{
Limitations in Energy Management Systems: A Case Study for Resilient Interconnected Microgrids
}

\author{
Leong Kit Gan, Member, IEEE, Akhtar Hussain, Student Member, IEEE, David A. Howey, Senior \\ Member, IEEE, and Hak-Man Kim, Senior Member, IEEE
}

\begin{abstract}
This paper explores the impact of EMS discrepancies that a microgrid may experience due to different time horizon and implementation environment in higher-level energy managements systems as compared to lower level control frameworks. These include time-shifting, magnitude deviation, and averaging effect of the renewables and load demand profiles. We use the resilient microgrid concept as a case study considering that the reliability of such system is crucial, especially during islanding mode. We demonstrate through experiment that the non-ideal effects naturally take place in a microgrid system, resulting a discrepancy between the measured and expected ideal battery state-of-charge. Accordingly, the resiliency of the microgrid may be affected if unplanned load shedding took place in order to not violate the lower limit of the battery state-of-charge. Allowing the battery to discharge at a higher depth may temporarily solve the problem, however, this comes at the expense of a higher rate of battery degradation. Instead, we proposed a power sharing scheme and by interconnecting the microgrids, the resiliency may be improved.
\end{abstract}

Index Terms - Battery, energy management system, microgrids, renewable energy, uncertainty.

\section{INTRODUCTION}

$\mathrm{T}$ HE concept of a microgrid has emerged as the fundamental technology representing a group of localised dispatchable generators, non-dispatchable generators, energy storage systems and load demand. Microgrids may or may not be connected to the main power grid. Recently, networked or interconnected microgrids are gaining more attention as they are known as the building blocks to realise the smart grid concept. Coordinating connected microgrids is non-trivial, especially when there are many nodes. Local and global energy management systems (EMS) are essential for tackling the challenges associated with managing and optimising the performance of microgrids.

Typically, an EMS operates at a lower bandwidth in comparison with the voltage, current and other control loops within power electronics converters. The former, also known as tertiary control [1], attempts to optimally balance supply and demand over a longer time interval. As part of an advanced

This work was supported by the Engineering and Physical Sciences Research Council under Grant EP/N034570/1, Korea Institute of Energy Technology Evaluation and Planning (KETEP) and the Ministry of Trade, Industry \& Energy (MOTIE) of the Republic of Korea (No. 20168530050030). (Corresponding authors: L. K. Gan and H.M. Kim). (email: leong.kit.gan@gmail.com).

L. K. Gan and D. A. Howey are with the Department of Engineering Science, University of Oxford, Oxford, OX1 3PJ, U.K.

A. Hussain and H.M. Kim are with Department of Electrical
EMS algorithm, it is common to predict renewable resources, load demands, and time of use tariff profiles subjected to uncertainties [2]. The EMS may individually or collectively consider the economic, technical or environmental constraints, depending on the types of load and services provided by the particular microgrid.

In the past, EMS research was notably focused on the formulation of constrained rule-based strategies [3, 4] whilst recent advances in machine learning techniques further motivate researchers in this field to work on predictive strategies coupled with optimisation [5-7], which is more realisable partly due to the presence of high computational power microprocessors. Nonetheless, the inherent characteristics and underlying assumptions made within an EMS may result in performance deviations between simulations of an idealised system, versus reality. For example, the battery state-of-charge (SoC) parameter which exists in both the EMS and the more localised battery management system (BMS) may not be computed consistently. There may include differences in sampling times, fidelity of the model used, and effects from real world phenomena such as resistive losses, communication latencies, sensor noise and measurement equipment calibration. Moreover, the uncertainties from a forecasting algorithm may lead to sub-optimal solution produced by the EMS.

In [8], an online-based EMS was implemented and studied. The overall architecture involved forecasting, scheduling, data acquisition, and interfacing. One interesting phenomena shown in this work is that the measured battery voltage may reach the saturation level earlier than expected, leading to a constantvoltage charging period, even before the battery SoC hits 100 $\%$. Hence, curtailment of renewable generation may take place at a local level, whereas the EMS might not be aware of this until the next update interval. In an islanded power system, it is recognised that SoC estimation errors may indeed cause power outages [9]. To mitigate this, the authors proposed a predictive pre-emptive non-critical load shedding approach whilst using empirically chosen uncertainty bounds in the SoC estimation.

It is also widely acknowledged that the uncertainties associated with a predictive algorithm are an important aspect when assessing the robustness of an EMS. A common way to emulate uncertainties is by adding a fixed noise term [10], which may be regarded as oversimplified. On the other hand, inappropriate selection of a predictive model may effect the performance of an EMS. In this context, a predictive model may be used for forecasting the renewable power and load demand, which is then used for model predictive control [5]. The authors 
in [11] have used a high fidelity model to validate the performance of an EMS and demonstrated that the battery SoC may deviate due to averaging effects and forecasting errors of the renewables and loads.

Demand-side management (DSM) is known as one of the tools which could bring cost savings for electricity consumers on microgrids by optimising their energy usage, for instance shifting energy consumption from peak to non-peak hours [12]. Conversely, the unplanned time shifting of generation and/or load due to the reasons discussed in the previous paragraphs, may bring adverse impacts in terms of electricity cost, and worse still, brownout in an electrical power supply system. Although trivial in the short term, the accumulated impact may be considerable in the long term.

In this paper, we investigate the limitations of an EMS including a simple power sharing scheme within an interconnected microgrid system, with each microgrid having a local battery system. Note that this study may be extended to other energy storage technologies. In this setup, any load shedding taking place will have a significant impact, especially the disconnection of critical loads during islanding mode. The objective of a resilient microgrid design is to mitigate the load shedding amount during major disruption events. This objective is achieved by preparing the microgrids via proactive scheduling before the occurrence of the event [13]. Similarly, survivability enhancement strategies are incorporated for improving the service reliability to loads, especially critical loads during the power outage events [14]. Generally, simplified battery models are utilised with a time step of 10 minutes to 1 hour for a resilient microgrid EMS [13-15]. In addition, various uncertainties associated with these EMSs, as discussed in previous paragraphs, are neglected. However, these uncertainties and simplifications may negatively influence the resilience of microgrids due to differences between the expected and actual amounts of available energy. Unplanned load shedding, especially on critical loads can result in both social costs and monetary losses. Therefore, the impact of these uncertainties needs to be analysed to better prepare microgrids for potential future events. In this context, this paper makes multiple contributions, which are summarised below:

- The factors contributing to the discrepancies of an EMS are modelled and analysed explicitly. These are mainly timeshifting, magnitude deviations and averaging inputs of renewable energy supplies and load demands

- The discrepancies of an EMS are demonstrated through experiments on laboratory scaled interconnected microgrids, which have realistic behaviour in terms of control, communication, measurements, and dynamics

- We demonstrated that the resiliency of an interconnected microgrid can be enhanced through a power sharing scheme which also considers the EMS uncertainties. The achieved resiliency improvement against non-connected microgrids is quantified

\section{MODELLING OF RESILIENT EMS}

Resiliency in power systems is defined as the ability of the power system to adopt and withstand low-probability high- impact events [13]. Among various solutions, microgrids are considered as a practical solution to enhance the resiliency of power systems due to their ability of islanding and potential for sustaining the penetration of renewables [16]. In order to utilise microgrids as an asset with high reliability, they need to be prepared for such events. Therefore, similar to [15], the resilience enhancement procedure proposed here is divided into two steps. In the first step, a proactive scheduling model is proposed to ensure feasible islanding and survivability of critical loads for next $n$ intervals after any major fault event. In the second step, the survivability of loads during an islanded period is considered by defining precedence between battery charging and feeding of non-critical loads. In addition, dynamic penalty costs are introduced for both critical and non-critical loads to potentially avoid unnecessary load shedding.

\section{A. Proactive Operation Model}

The proactive operation model is applied before the event occurs, i.e. during grid-connected mode. In this mode, a scheduling horizon, $T$ of 24-hours is considered with a sampling time, $t$ of 1 minute, except for the averaging and combined effects in Section III D and E. An event in this study refers to major outages which are caused by natural disasters. Various extreme weather conditions such as floods, tsunamis, hurricanes, earthquakes, and heavy rains, etc. have caused major outages in the recent years. The severity and frequency of these events are expected to increase in the near future due to climate change [17]. The resilience-oriented operation scheme considered in this study focuses on extreme weather events. Resilience-oriented operation schemes are conservative and they may result in increased operation cost. Therefore, such schemes are not recommended to be utilised throughout the year. In case of extreme-weather events, initial warnings about the events are issued by the local meteorological agencies [18]. The proposed proactive operation scheme can be activated once the initial warnings are issued.

The base model of the proposed resilience-oriented operation scheme is similar to [15]; therefore, only a compact form of the base model is shown in this study. For detailed mathematical models of all the microgrid components, refer to [15]. Equation (1) shows that the objective of the base model is to minimise the operation cost of the microgrid during grid-connected mode while satisfying some equality and inequality constraints. In (1), c represents the price of power traded between the microgrids and the utility grid and generation cost of dispatchable generators. $\mathbf{x}$ represents the amount of power traded between microgrids and the utility grid, amount of power transferred among the microgrids of the network, and power generated by dispatchable units. Time-of-use market price signals are utilised in this study for the proactive operation phase. In (2), $\mathbf{F x} \leq \mathbf{g}$ represents the constraints for trading power among the microgrids, trading power between microgrids and the utility grid, and generation limits of dispatchable generators. $\mathbf{F}$ is a matrix, which represents the efficiency of the power lines (lines connecting microgrids with the utility grid and lines interconnecting microgrids) and it contains a unity row vector for dispatchable generators. Matrix $\mathrm{g}$ represents the capacity (power limit) of the power lines and 
generation bounds of dispatchable generators. $\quad \mathbf{R y} \leq \mathbf{h}$ represents the constraints to improve resiliency, where $\mathbf{y}$ indicates the variables associated with energy storage elements, i.e. charging/ discharging constraints and SoC constraints. In case of charging/discharging constraints, $\mathbf{y}, \mathbf{R}$, and $\mathbf{h}$ respectively represent the charging/discharging power, efficiency, and charging/discharging rate of the energy storage elements. In case of additional resiliency constraints, $\mathbf{y}$ and $\mathbf{h}$ respectively represent the left and right sides of equation (4) and $\mathbf{R}$ is a unity vector. In contrast to [15], where only feasible islanding is considered, additional constraints for battery SoC are also incorporated in the operation model of this study, as shown in (2). The inequality sign can be reversed by multiplying both sides of the equation with -1 . Equation (3) computes the net critical load and is incorporated in the righthand side of equation (4). Finally, $\mathbf{G y}=\mathbf{r}-\mathbf{P x}$ represents the power balancing constraint containing both power trading and power generation ( $\mathbf{x}$ ) and battery ( $\mathbf{y}$ ) variables, where, $\mathbf{G}$ is the charging/ discharging efficiency of the energy storage elements of the microgrid, $\mathbf{r}$ is the net power of the microgrid and $\mathbf{P}$ is the efficiency of the power lines, i.e. lines for interconnecting the microgrids and lines for connecting microgrids with the utility grid. The values of vector $\mathbf{P}$ are identical to the values of first row of matrix $\mathbf{F}$. The additional constraints for battery SoC in each microgrid $m$ are realised via Equations (3), (4).

$\min \mathbf{c}^{\mathrm{T}} \mathbf{x}$

subject to:

$$
\begin{aligned}
& \mathbf{F x} \leq \mathbf{g}, \mathbf{R y} \leq \mathbf{h}, \mathbf{G} \mathbf{y}=\mathbf{r}-\mathbf{P x} \\
& N L_{m, t}^{c}=\left\{\begin{array}{ll}
0 \quad \text { if } P_{m, t}^{c l}<P_{m, t}^{r} \\
\left(P_{m, t}^{c l}-P_{m, t}^{r}\right) \cdot\left(100 / P_{m}^{c a p}\right) & \text { else }
\end{array} \quad \forall m, t\right.
\end{aligned}
$$

The net critical load amount at each interval $t$ for microgrid $m$ $\left(N L_{m, t}^{c}\right)$ is determined using the information of critical load in watt-hour $\left(P_{m, t}^{c l}\right)$ and available renewable energy $\left(P_{m, t}^{r}\right)$ as given by (3). The net critical load is normalized with the battery capacity $\left(P_{m}^{c a p}\right)$ and is transformed into percentage for unifying the units with SoC. The information of net critical load is used to update the lower SoC bounds of the battery in microgrid $m$ ( $S o C_{m}^{\min }$ ), as given by (4). A resilience-oriented lower bound ( $\operatorname{SoC}_{n, m, t}^{r e s}$ ) is defined for battery units to assure the survivability of critical loads for next $n$ intervals after the occurrence of an event.

The $n$ value for microgrid $m$ depends on the amount of critical load, renewable power, and the battery capacity within the microgrid. The maximum $n$ value for the entire scheduling horizon is selected considering the parameters mentionedabove. In this study, the maximum feasible $n$ is 120 intervals, i.e. 2-hours.

\section{B. Survivability-Oriented Operation Model}

The survivability-oriented operation scheme is applied after an event occurs, i.e. when the system is in islanded mode. During the emergency period, the operation horizon is from the event occurrence time, $t_{\mathrm{e}}$ until the end of the day, $T$. The event time can be estimated using the fragility curves information of the particular parameter(s) representing the event $[19,20]$. However, in this study, the event time is assumed to be known due to the focus of this study on analysing the limitations of EMS.

The objective of the operation model is to maximise the survivability of power supply to the loads, especially the critical loads. The compact form of the survivability-oriented operation model is given by the Equations (5)-(10). In Equation (5), C represents the penalty cost for shedding loads and generation cost of dispatchable units. s represents the amount of load shed, amount of power generated by dispatchable units, and amount of power transferred among the microgrids of the network. The dynamic penalty cost for critical and non-critical loads in Equation (5) can be computed using Equations (7) and (8). In (6), As $\leq \mathbf{b}$ represents the constraints for critical and noncritical load shedding and dispatchable generation limits. Matrix b represents the amount of critical and non-critical loads in the microgrids and generation bounds of dispatchable generators. A is a unity matrix. Gy $\leq \mathbf{d}$ represents the constraints related to battery units with $\mathbf{G}$ as the charging/discharging efficiency vector and $\mathbf{d}$ is the charging/discharging rates vector. $\mathbf{G y}=\mathbf{u}-\mathbf{Q s}$ represents the power balance in the islanded mode, where, $\mathbf{u}$ is the net renewable power of the microgrid in islanded mode and $\mathbf{Q}$ is the line efficiency of lines interconnecting microgrids for power transfer among microgrids and is a unity matrix for load shedding. In contrast to [15], where a flat penalty cost is used for critical and non-critical loads, a dynamic penalty cost is introduced in this paper. The dynamic penalty cost, as defined in (7)-(10), shifts the load shedding towards the end of the scheduling horizon. This can potentially avoid unnecessary load shedding if the event is cleared before the end of the scheduling horizon.

$\min \mathbf{C}^{\mathrm{T}} \mathbf{s}$

Subject to:

$$
\begin{array}{cr}
\mathbf{A s} \leq \mathbf{b}, \mathbf{G y} \leq \mathbf{d}, \mathbf{G y}=\mathbf{u}-\mathbf{Q s} & \\
P C_{t}^{c, p e n}=P C^{c f, p e n}-\alpha^{c} . t & \forall t \in\left[t_{e}, T\right] \\
P C_{t}^{n, p e n}=P C^{n f, p e n}-\alpha^{n} . t & \forall t \in\left[t_{e}, T\right] \\
\max \left[\mathbf{p c} \mathbf{c}^{\mathbf{n}, \mathbf{p e n}}\right]<\min \left[\mathbf{p c ^ { c , p e n }}\right] & \\
P C^{c f, p e n}>P C^{n f, p e n}, \alpha^{c} \geq \alpha^{n} &
\end{array}
$$

The dynamic penalty cost of critical loads for each time interval $t\left(P C_{t}^{c, p e n}\right)$ can be computed using the fixed penalty cost ( $\left.P C^{c f, p e n}\right)$ and decrement factor $\left(\alpha^{c}\right)$, given by $(7)$. Variable $t$ is the time step of the optimisation model, which could be any uniform interval of time. Similarly, the dynamic penalty cost of non-critical loads can be computed using (8). In Equations (7) and (8), $t_{e}$ represents the event occurrence time i.e. beginning of the islanded operation and $T$ represents the end of the scheduling horizon i.e. end of the day for this study. The 
relation between the penalty costs of critical and non-critical loads is given by (9). Finally, the relation between fixed penalty cost and decrement factor is given by (10). The fixed penalty cost and the decrement factor introduced in this study are relative to each other. Therefore, any values satisfying the constraints defined in Equations (9) and (10) will give the same operation results. If the system consists of dispatchable generators, the fixed penalty cost of non-critical loads should be higher than the generation cost of the most expensive dispatchable unit. This ensures the precedence of service reliability over the operation cost during the emergency period, i.e. islanded mode.

A resilience index $\left(R I_{m, t}\right)$ is formulated to quantify the impact of interconnecting the microgrids on the resilience of the microgrid network. The recovered load $\left(P_{m, t, \rho_{n}}^{r l}\right)$ and actual load $\left(P_{m, t, \rho_{n}}^{a l}\right)$ parameters are used to compute the resilience index for each microgrid $m$, as shown in (11). Where, $\rho_{n}$ is the priority index for $n^{\text {th }}$ level load and $N$ is the total number of load levels in the network. The value of $\rho_{n}$ for critical loads is higher that of non-critical loads. The maximum $\left(R I_{\mathrm{m}, \max }\right)$ and normalized $\left(\overline{R I}_{\mathrm{m}, \mathrm{t}}\right)$ values of the resilience index are computed using (12). Finally, the acceptable bounds of the resilience index for microgrid $m$ are computed using (13).

The objective of this resilience index is to evaluate the microgrid's performance under different scenarios. These include individual microgrid operation, interconnected microgrid operation, and the hardware test of the interconnected microgrid case. The index indicates the amount of load shed (both critical and non-critical) in the microgrid network. Shedding of higher priority loads results in lower index values compared to the lower priority loads, as shown in (11). The index is normalised as shown in (12). The acceptable bound in Equation (13) indicates the minimal acceptable resilience index for the survival of all the critical loads.

$$
\begin{aligned}
& R I_{m, t_{e}}=\left(\sum_{n \in N} \rho_{n} \cdot \frac{P_{m, t_{e}, \rho_{n}}^{r l}}{P_{m, t_{e}, \rho_{n}}^{a l}}\right) / N \\
& R I_{\mathrm{m}, \max }=\sum_{n \in N} \rho_{n} / N, \overline{R I}_{\mathrm{m}, t_{e}}=R I_{\mathrm{m}, t_{e}} / R I_{\mathrm{m}, \max } \forall n \in N, t_{e} \in T \\
& R I_{\mathrm{m}, \mathrm{acc}} \in\left[1 /\left(N \cdot R I_{\mathrm{m}, \max }\right), 1\right] \quad \forall n \in N, t_{e} \in T
\end{aligned}
$$

In general, methods to solve optimisation problems are based on the type of search space and objective function. The simplest method is the Linear Programming that solves only a linear objective function subject to linear equality or inequality constraints [21]. On the contrary, Nonlinear Programming is aimed for solving nonlinear objective functions and constraints. Where problems involve uncertainty, Stochastic Programming is a suitable framework that take advantage of probability distributions of various variables. Computational-intelligence techniques such as Genetic Algorithm [22] and Particle Swarm Optimisation [23] have been proposed to determine global optimum solutions, however, at the expense of high computational effort [24]. The choice between the abovementioned options is associated with the trade-off between accuracy (more optimal solutions) and computational time.

Given the fact that components in a microgrid such as wind

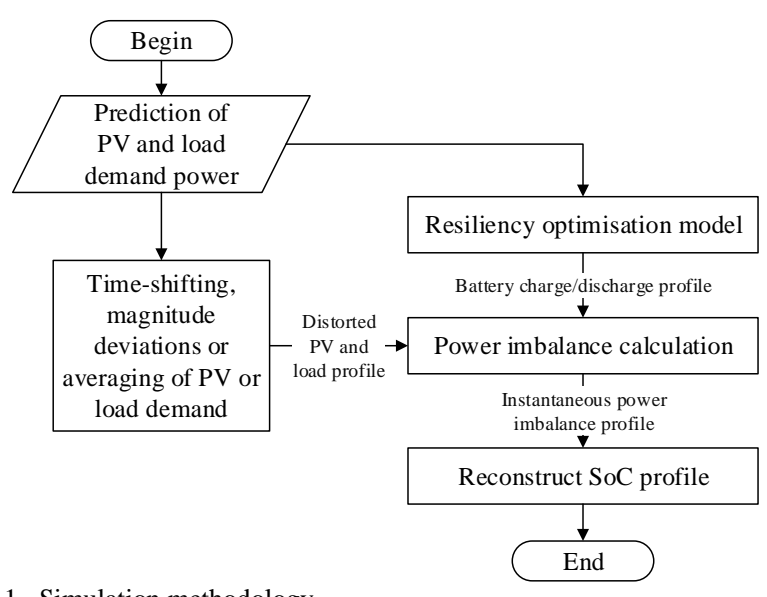

Fig. 1. Simulation methodology

turbines, solar PV, batteries, diesel generators, power electronic systems etc, are non-linear in nature, coupling with the nonlinearity of a power system, non-linear optimsers seem to be the ideal candidates for solving real interconnected microgrid problems. The considerations and literature review of the applications of optimisation techniques for microgrids can be referred in [25]. Whilst non-linear models provide better representation, linearised model are often being adopted to reduce computational effort. Generally, piecewise linearisation of dispatchable generators, lossless or linear transmission line's power losses, constant charging/discharging efficiency of energy storage elements, and linear losses of converters are considered whilst formulating an EMS. Since the objective of this research is to demonstrate and quantify the impact of low fidelity models (implemented in EMS) on the resiliency of real microgrids, linearised EMS model is considered in our work. A mixed integer linear programme (MILP) is selected and we adopted the commercial CPLEX solver from IBM. The limitations are thus uncovered through experimental measurements where nonlinearities of the real microgrid components are inherited.

\section{CAUSES OF EMS UNCERTAINTIES AND THEIR IMPACT ON BATTERY SOC}

This section investigates several factors which can lead to discrepancies in the variables used within an EMS, compared to reality. In particular, we examine time shifting, magnitude deviations, averaging effects, and a combination of these for photovoltaic (PV) and load profiles respectively. A network of three microgrids is considered in this paper. However, for the sake of visualisation, the EMS discrepancies on a single microgrid is analysed in this section. In Section IV, a whole network is analysed and corrective measures are suggested.

\section{A. Simulation Methodology}

The simulation methodology is shown in Fig. 1. Feeding the predicted PV and load profiles into the optimisation model which was described in Section II, the optimised battery charging/discharging sequence and its corresponding SoC profile are determined. Hereafter, we refer to this as the ideal EMS case.

At every instance (in seconds), the power imbalance ( $P_{m, t}^{\text {error }}$ ) within the system is calculated using equation (14). The power 


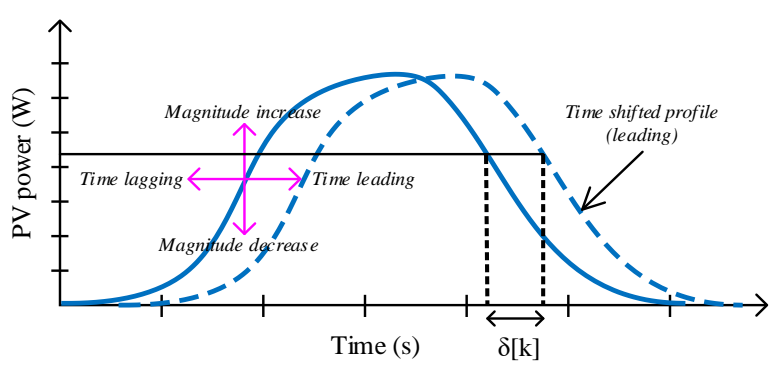

Fig. 2. An illustration of time advancing the PV profile
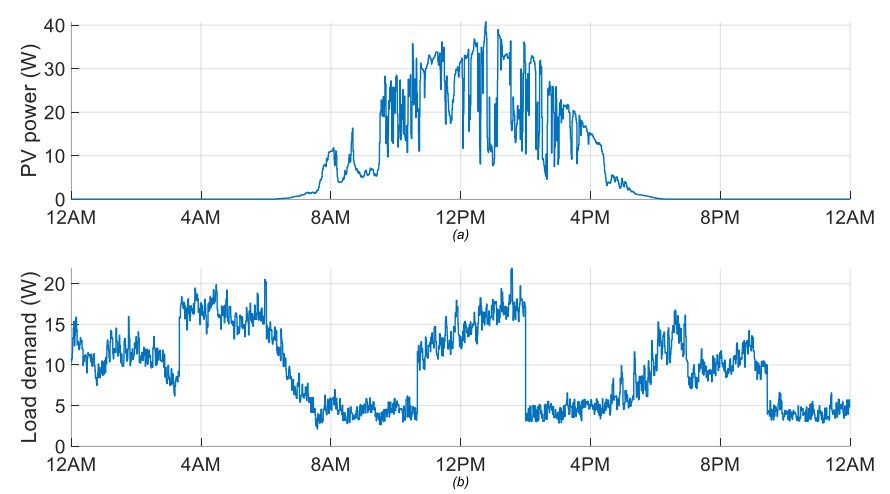

Fig. 3. PV and load demand profile input for optimisation model

charged/discharged to/from the batteries $\left(P_{m, t}^{b a t}\right)$, power traded with the utility grid $\left(P_{m, t}^{\text {grid }}\right)$, and power transferred between microgrid $m\left(P_{m, t}^{\text {trans }}\right)$ and other microgrids of the network along with modified profiles of PV $\left(P_{m, t}^{p v^{\prime}}\right)$ and load $\left(P_{m, t}^{\text {load }}\right)$ are used to compute the power imbalance.

$$
P_{m, t}^{\text {error }}=P_{m, t}^{p v^{\prime}}+P_{m, t}^{\text {bat }}+P_{m, t}^{\text {grid }}+P_{m, t}^{\text {trans }}-P_{m, t}^{\text {load }}
$$

For the ideal EMS case, power imbalance is null because the power balance constraint in (2) must be met at all time steps during optimisation process. However, when either the PV or load profile is altered (to mimic uncertainties), and whilst using the original battery charge/discharge profile, power surplus or deficit situations may occur. Assuming that the battery is supplying/absorbing the difference, the $\mathrm{SoC}$ profile may therefore deviate from the ideal case. The power imbalance information is then used to reconstruct the deviated SoC profile.

Fig. 2 illustrates an example when advancing the PV profile with a time step of $\delta[\mathrm{k}]$, which is referred as the time-leading case. To represent magnitude deviation, the PV or load profile is shifted in the y-axis. Due to computational constraints and the availability of electricity price updates, many EMS systems re-run their optimisation relatively infrequently (for example half-hourly). This requires averaging of the measured inputs (e.g. PV power and load demand in this case) according to the required sampling time. Hypothetically, this may contribute to EMS discrepancies, especially during sub-hourly intervals. In this work, we considered 1-minute as the highest resolution for both PV and load profiles. However, their fluctuations are most likely to occur at higher rates in reality, potentially bringing more uncertainties to the EMS than we may find here.

Fig. 3 shows the PV power and load profiles for the following case studies in this section. The solar irradiance time series data obtained from National Renewable Energy Laboratory (1minute resolution historical data) was used in this work,

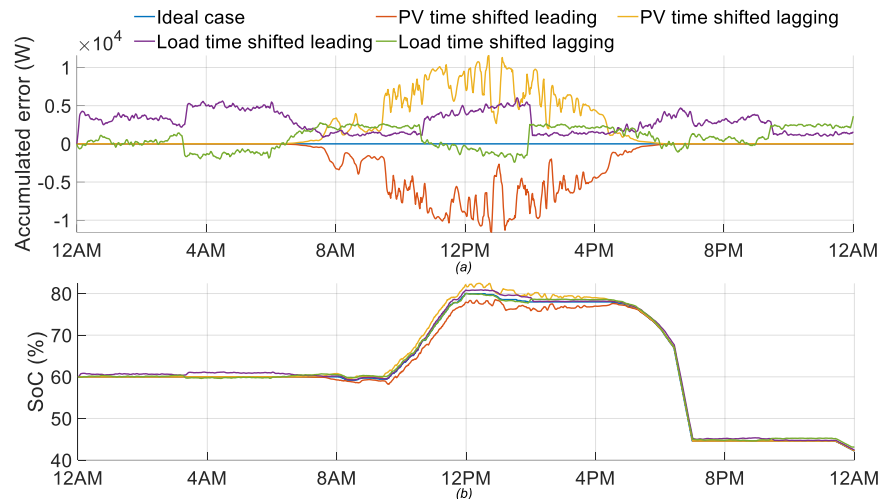

Fig. 4. (a) Power imbalance error profile (b) SoC profile for PV and load demand time advancing (leading) and delay (lagging) of 5 minutes

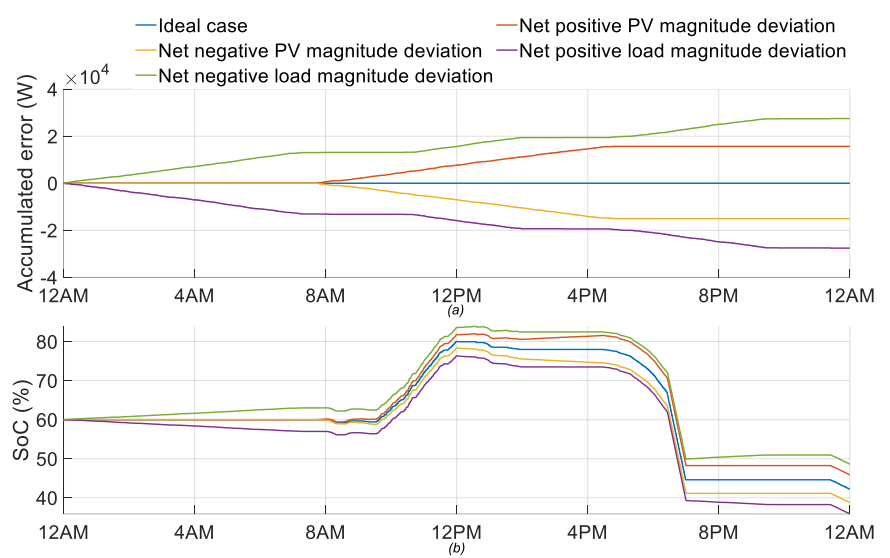

Fig. 5. (a) Power imbalance error profile (b) SoC profile for PV and load demand magnitude deviations

although ideally, the availability of extreme weather data may reflect a more accurate scenario. As measured load data were not freely available, this work adopted an open-source bottomup domestic load model [26] which has been qualitatively validated [27]. The load model synthesised the load profile used in this work. In the following studies, the profiles in Fig.3 represents the true values. They are altered according to the considered factors, i.e., time shifts, magnitude deviation or averaging. It is also worth mentioning that we have downscaled the power rating to match our experimental test-rig (described in section IV-A).

\section{B. Time Leading and Lagging}

Fig. 4 demonstrates the results when the PV and load profiles are advanced or delayed for 5 minutes, with the top figure showing the accumulated power imbalance whilst the bottom figure illustrates the deviated SoC profiles. For the ideal case, the power imbalance error is zero and the SoC stays between the $30 \%$ and $80 \%$ limits.

Several trends can be noticed from the plots. First, the characteristics of power imbalance for shifting the PV and load demands are dissimilar. Shifting the former will only deviate the battery $\mathrm{SoC}$ when the PV system starts generating power. Hence, the SoC is deviated only during the daytime. On the contrary, the load demand causes SoC deviation throughout the day and night. Secondly, if the PV profile has a constant delay (lagging case) of 5 minutes, the battery needs to supply the deficit and hence, the battery SoC overshoots the 80\% upper 

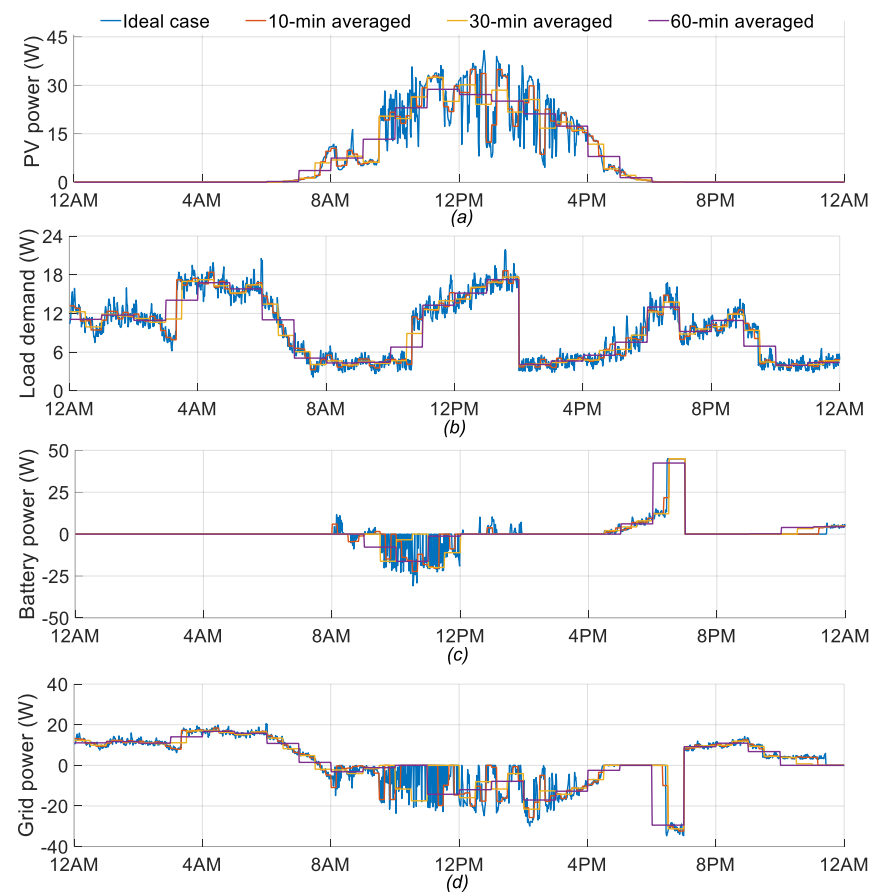

Fig. 6. 10-min, 30-min and 60-min averaging of (a) PV (b) load demand (c) battery power (d) grid power profiles
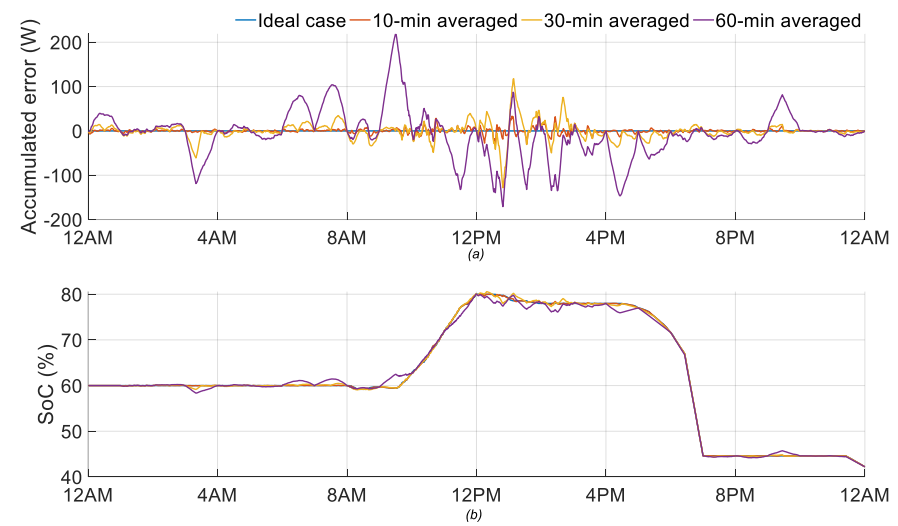

Fig. 7. (a) Power imbalance error profile (b) SoC profile for different averaged sampling time

limit around the mid-day. But when the solar irradiance comes earlier than the predicted profile (leading case), the battery would be slightly under-used especially around mid-day.

Finally, since the battery SoC deviation is a function of the power imbalance magnitude, the highest discrepancy occurring at mid-day. In other words, the EMS discrepancy may be highly influenced by inaccurate timing estimation of the PV power compared to the load demand, mainly due to the higher peak value of the former.

\section{Magnitude Deviation}

Here, we study the extent to which the battery SoC may be affected when renewable resources or load demands are overor under-predicted for an extended period. We randomised the PV and load profiles with a vector of uniformly distributed random numbers. The upper limit of the random numbers is assigned with a value of $10 \%$ above the mean value (zero in this case) to represent over-prediction of PV or load, and vice versa.

Fig. 5 shows the simulation results. It is observed that the power imbalance occurs continuously when adding magnitude
TABLE I

PARAMETERS FOR COMBINED EFFECTS

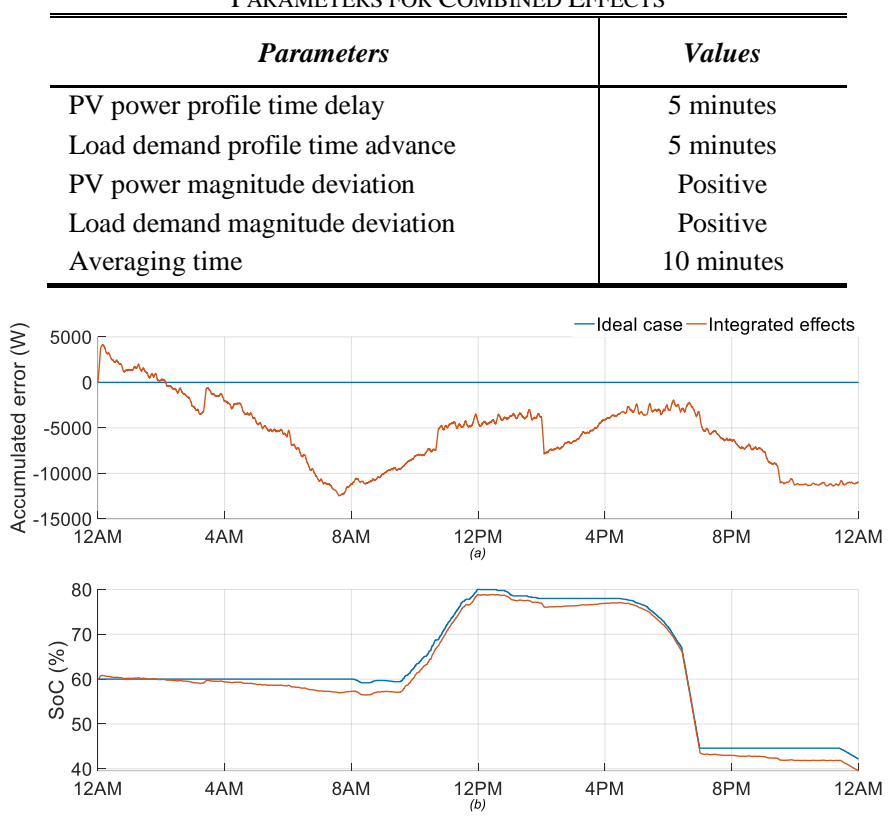

Fig. 8. (a) Power imbalance error profile (b) SoC profile for integrated effects

uncertainty to the load demand, whereas the PV system only starts to generate power at about 8 a.m. and hence, the power imbalance begins at that moment. In general, the battery SoC may deviate significantly from its expected profile (ideal case). In addition, the magnitude deviation may have a stronger influence on the battery SoC deviation compared to the timing uncertainties (Fig. 4). However, this is still largely dependent on the scale of the magnitude deviation.

\section{Averaging}

Fig. 6 shows the comparison between 1-minute (ideal case), 10-minutes, 30-minutes and 1-hour averaging on PV power, load demand, battery charge/discharge power and power trading with the utility grid. In this study, the power imbalance for 10-minutes, 30-minutes and 1-hour averaging is computed against the 1-minute sampling time. As expected, the power imbalance occurred within the sub-hourly intervals and lower update rates for the EMS indeed induce more volatility in the battery SoC (see Fig. 7a). Interestingly, the ideal case's battery $\mathrm{SoC}$ is the averaged profile of the more fluctuating hourly averaged SoC profile. This study implies that the averaging approaches adopted when optimising a power flow operation to predict the battery SoC, seem to be reasonable for long-term case studies, although the instantaneous actual SOCs may become out-of-bounds if the EMS updates are infrequent.

\section{E. Combined Effects}

Whilst the fundamental understanding of EMS uncertainties is developed through the individual factors previously described, combining them to mimic a more realistic scenario is presented in this section. Besides the averaging effect, we consider delay and net positive magnitude deviation in PV power, in addition to time-leading and positive magnitude shifts for load demand. The parameters are shown in Table I. 


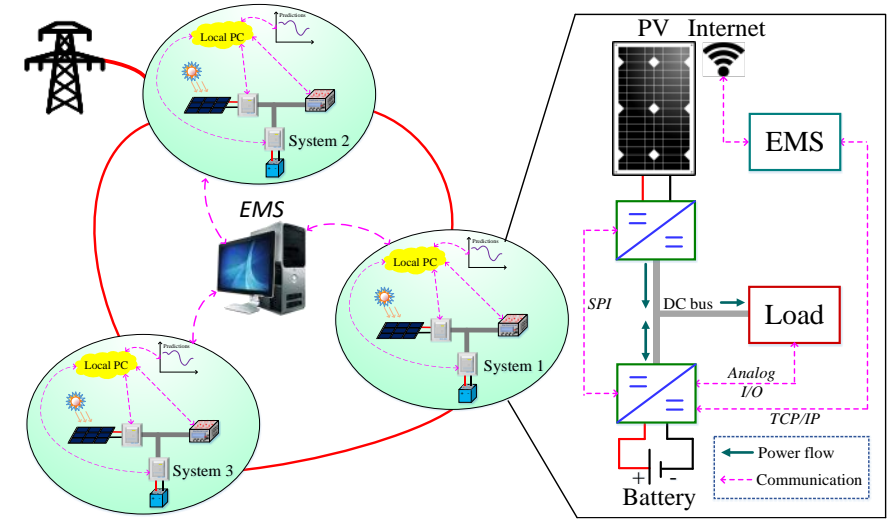

Fig. 9. Block diagram of the laboratory-scaled networked microgrids

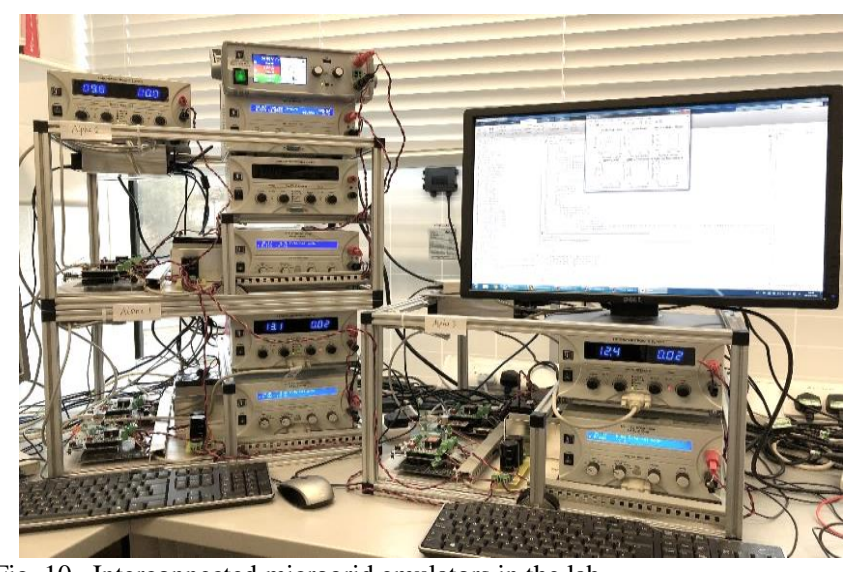

Fig. 10. Interconnected microgrid emulators in the lab

Fig. 8 demonstrates the results with the top plot showing the power imbalance whilst the bottom plot corresponds to the comparison of battery SoC between the ideal case vs. the combined approach. The overall trend is that the battery $\mathrm{SoC}$ is less predictable compared to the previous cases. In this case, the battery $\mathrm{SoC}$ rose above the expected level until about 2 a.m., however, always in shortage beyond that. The highest discrepancy occurred just before 8 a.m., which accounted to about $3 \%$ battery SoC.

\section{EVALUATION OF UNCERTAINTIES IN INTERCONNECTED MICROGRIDS}

In this section, we aim to understand the resiliency aspect of a microgrid system by interconnecting several smaller neighbouring microgrids. First, a brief description of our laboratory-scaled interconnected microgrids is given. A case study is performed to compare and contrast the ideal EMS against the experimental results. In particular, we used the battery $\mathrm{SoC}$ and power transfer between the microgrids and the utility grid as the performance indicators for evaluation. Finally, we present a simple power sharing scheme which is suitable for use with interconnected microgrids.

\section{A. Laboratory-scaled interconnected microgrids}

In this work, we used three interconnected microgrid emulators to demonstrate some of the uncertainties discussed in the previous sections. The microgrid system block diagram is shown in Fig. 9. The microgrid emulators can communicate with each other via TCP/IP, enabling development and demonstrations of distributed forecasting, control, and
TABLE II

EXPERIMENTAL SPECIFICATIONS

\begin{tabular}{l|c}
\hline \multicolumn{1}{c|}{ Parameters } & Values \\
\hline Sampling time & 1 minute \\
Battery SoC boundary & $30 \leq \mathrm{SoC} \leq 80$ \\
Battery capacity & $120 \mathrm{Wh}$ \\
Battery charging efficiency & $95 \%$ \\
Battery discharging efficiency & $96 \%$ \\
Maximum and minimum battery power & $\pm 45 \mathrm{~W}$ \\
Maximum and minimum grid power & $\pm 131.25 \mathrm{~W}$ \\
Transmission line resistance & $0.01 \Omega$ \\
Critical and non-critical load ratio & $1: 3$ \\
Survivability intervals, $n$ & $120(2 \mathrm{hours})$ \\
Fixed penalty cost (Critical, non-critical) & $£ / \mathrm{kWh}(5,2)$ \\
Decrement factor (Critical, non-critical) & $(0.001,0.0007)$ \\
\hline
\end{tabular}

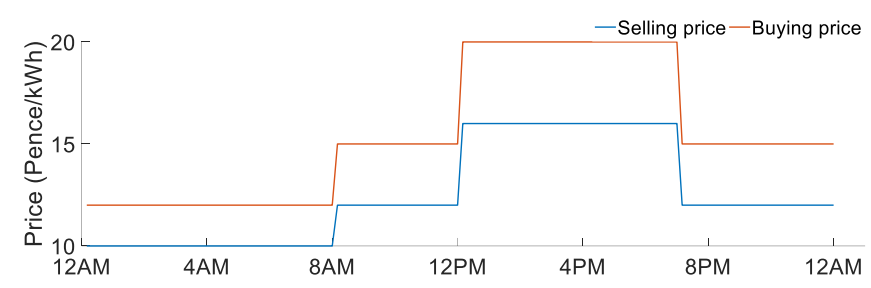

Fig. 11. Time-of-use market price signal for test network

optimisation. Although the system is a scaled-down microgrid system, it nonetheless is realistic in terms of control interfaces, measurements, and dynamics, and therefore provides a valuable insight into EMS implementation which cannot be obtained through simulations alone. For simplicity, we adopted a centralised EMS approach. In this configuration, the utility grid is connected to microgrid 2 whilst the rest are power linked with each other with a ring topology.

The adopted microgrid configuration is a dc system, which has the advantages of not requiring synchronisation, frequency and phase control devices. The PV emulator and lead-acid battery are connected to the common dc bus via bespoke bidirectional half bridge dc-dc converters. For simplicity, a power supply and an electronic load are used to emulate the utility grid. The power supply is programmed to control the voltage of the common dc bus and it supplies the power deficit within the microgrid system. In terms of software structure, a low-level algorithm which is implemented in the ARM-based microcontroller (STM32F401RET6) executes the control logic and instantiates communication links between the EMS algorithm and hardware. The low-level logic mainly controls the dc-dc converters, provides a current reference for the electronic load, implements the battery management system and protects the microgrid. The next layer is equipped with Matlab software which provides a platform for EMS deployment. More details on the hardware and software implementation of the microgrid can be found in [28].

\section{B. Experimental Demonstration of EMS Discrepancies}

A case study is formulated to evaluate the EMS discrepancies. The PV and load profiles (1-minute resolution) were averaged over 10-minute intervals and were fed as inputs to the optimisation algorithm. The 1-minute resolution profiles were used as control inputs for the PV emulator and electronic load. In addition, the battery charge/discharge profiles which were computed by the EMS were used as set-points for the 


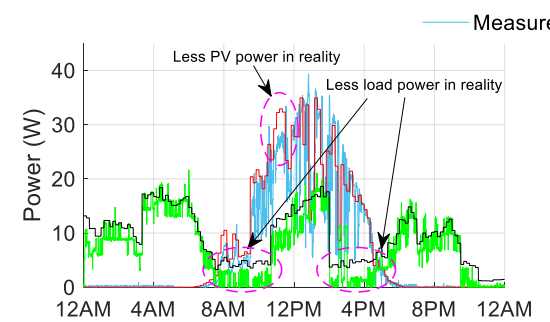

(a)

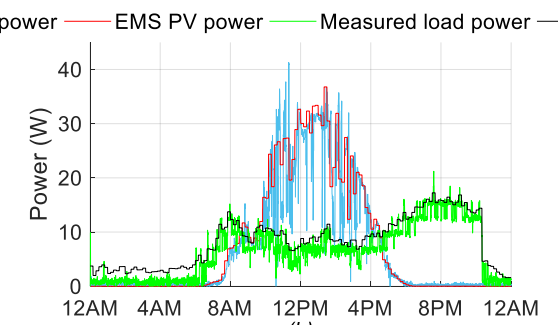

(b)

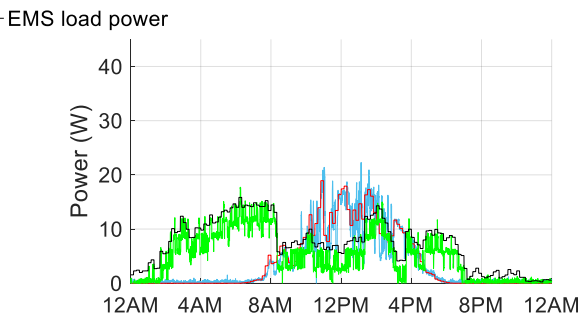

(c)
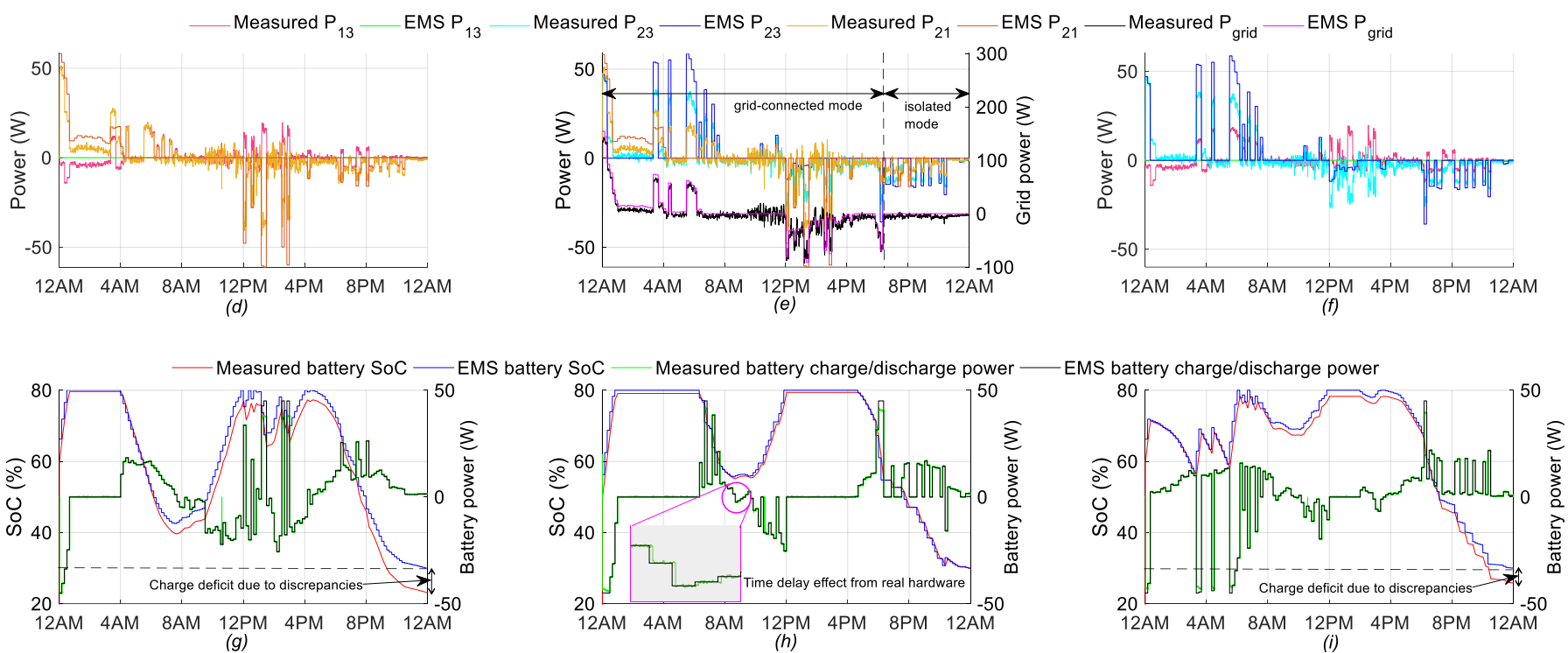

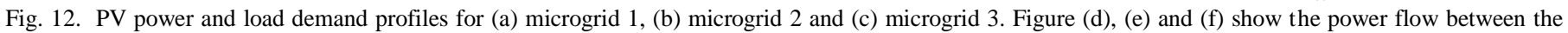
microgrids. The battery charge/discharge and its corresponding SoC profiles for (g) microgrid 1, (h) microgrid 2 and (i) microgrid 3

battery power converter. Note that in this case, to investigate resiliency, we set a disruption event at 6:20 p.m. From this moment onwards, the microgrids were disconnected from the utility grid and operated in isolation. The experimental specifications of the test system are tabulated in Table II and the time-of-use market price signal is shown in Fig. 11.

Fig. 12 shows the experimental measurements along with EMS estimates. More fluctuations can be observed in the measured PV power and load demand profiles compared to their corresponding 10-minute averaged EMS expected profiles, as shown in Fig. 12a, b and c. Moreover, substantial discrepancies between the ideal and measured profiles are noticed, as pointed out in Fig. 12a. These relate to the magnitude deviation effects which were discussed in Section III C. In this setup, the deviations may be attributed to the maximum power point tracking algorithm of the PV converter performing poorly at that particular interval whilst the electronic load is less sensitive to control signals at low load level.

Fig. 12d, e and $\mathrm{f}$ show the power flow among the microgrids, with microgrid 2 being the point of common coupling to the main grid. Electricity import mostly occurs earlier in the day, when the electricity price is relatively cheaper than in the evening. More power transfer can be observed between microgrids 2 and 3 , and between microgrids 2 and 1 , due to connection of microgrid 2 with the utility grid. However, minimal power flow takes place between microgrids 1 and 3 due to their similar load and PV profiles.

Fig $12 \mathrm{~g}, \mathrm{~h}$ and i display the battery charge/discharge profiles and their corresponding SoC curves, in both experimental measurement and EMS simulation results. At every second, the battery $\mathrm{SoC}$ is updated using the local microcontroller whilst the EMS calculates the SoC only every 10 -minutes. The batteries began to charge when there was generation surplus from the PV system, but between 12 p.m. and 4 p.m., any excess amongst the microgrids was exported to the main grid.

The measured battery SoC deviated from its ideal profile, visibly clearer in the case of microgrid 1 and 3 compared to microgrid 2. Towards the end of the day, the battery SoC in microgrids 1 and 3 dropped beyond the lower limit and this is more detrimental in terms of battery lifetime for the lead-acid cells used here [29]. If the $\mathrm{SoC}$ were to be maintained at $30 \%$ (lower limit), additional load shedding would be required and hence, the resiliency of the microgrid would be compromised. One of the contributors to this discrepancy, besides the uncertainties described above, is the presence of losses in the experimental setup. Since microgrid 2 is directly connected to the main grid which controls the bus voltage, the voltage drop within that microgrid is minimal compared to the downstream microgrids 1 and 3. Due to the higher voltage drops experienced by the latter, the batteries must provide more current to achieve the same power output. For a day's operation, the battery SoC may be $5 \%$ to $7 \%$ lower than expected, depending on the distance of the particular microgrid from the slack bus. This is significant over a longer timescale and should be considered by an EMS in the field.

Finally, the time delay effects from the hardware were also successfully captured, as highlighted in Fig. 12h. The hardware took about 1 minute to respond to the EMS request and although 


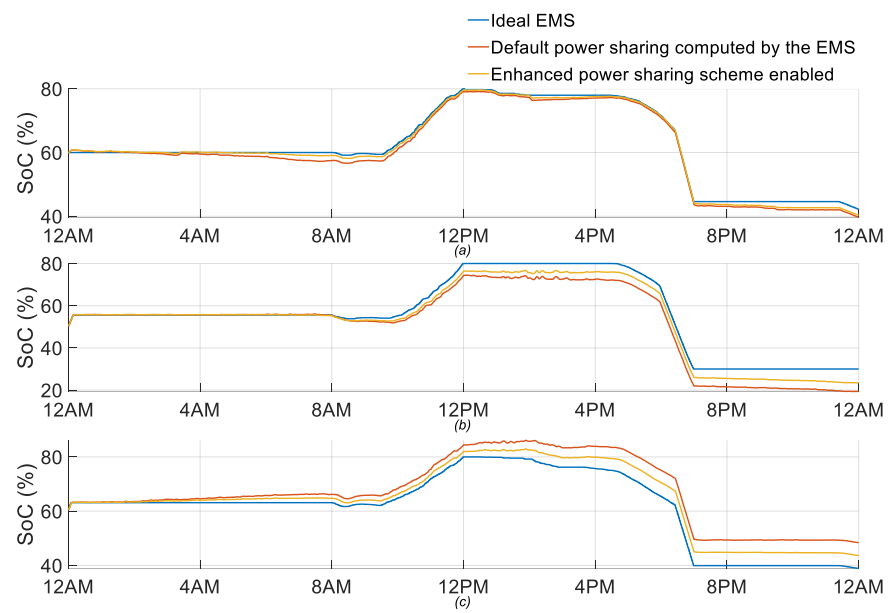

Fig. 13. SoC profiles for (a) microgrid 1 (b) microgrid 2 and (c) microgrid 3 before and after enhanced power sharing scheme enabled

TABLE III

RESILIENCE INDEX VALUES FOR DIFFERENT TEST CASES

\begin{tabular}{lll|lll|lll}
\hline \multicolumn{3}{c}{ Interconnected case } & \multicolumn{3}{c}{ Unconnected case } & \multicolumn{3}{c}{ Hardware test case } \\
\hline MG1 & MG2 & MG3 & MG1 & MG2 & MG3 & MG1 & MG2 & MG3 \\
\hline 1.00 & 0.63 & 1.00 & 1.00 & 0.63 & 1.00 & 0.00 & 0.63 & 1.00 \\
1.00 & 0.63 & 1.00 & 1.00 & 0.63 & 1.00 & 0.00 & 0.63 & 0.56 \\
1.00 & 0.63 & 1.00 & 1.00 & 0.63 & 1.00 & 0.00 & 0.63 & 0.00 \\
0.63 & 0.63 & 0.63 & 1.00 & 0.63 & 1.00 & 0.00 & 0.63 & 0.00 \\
0.63 & 0.63 & 0.63 & 1.00 & 0.63 & 1.00 & 0.00 & 0.63 & 0.00 \\
0.63 & 0.63 & 0.63 & 1.00 & 0.63 & 1.00 & 0.00 & 0.63 & 0.00 \\
0.63 & 0.63 & 0.63 & 1.00 & 0.63 & 1.00 & 0.00 & 0.63 & 0.00 \\
0.63 & 0.63 & 0.63 & 1.00 & 0.63 & 1.00 & 0.00 & 0.63 & 0.00 \\
0.63 & 0.63 & 0.63 & 1.00 & 0.11 & 1.00 & 0.00 & 0.63 & 0.00 \\
0.63 & 0.63 & 0.63 & 1.00 & 0.00 & 1.00 & 0.00 & 0.63 & 0.00 \\
0.63 & 0.63 & 0.63 & 1.00 & 0.00 & 1.00 & 0.00 & 0.63 & 0.00 \\
0.63 & 0.63 & 0.63 & 1.00 & 0.00 & 1.00 & 0.00 & 0.44 & 0.00 \\
\hline
\end{tabular}

it is beyond the scope of this work, the possible stability implications of this are worth investigating.

\section{Power Sharing Control for Interconnected Microgrids for mitigating EMS Discrepancies}

The question whether to physically link neighbouring microgrids may be answered by considering the additional value gained by this enabling, in theory, a more reliable system. This may be quantified by calculating the reduced amount of load shedding during islanded mode.

Here, we propose a simple power sharing scheme where the microgrids aim to reduce their discrepancies between the actual batteries SoC and the ideal EMS pre-computed SoC. At every time step, the power imbalance is assessed among all the microgrids. If there is power surplus in at least one of the microgrids and the remaining is/are experiencing power deficit, the power sharing control is enabled. Then, the net difference between the total power surplus and deficit is evaluated. If the net value is positive, it means that the excess can fully compensate for the deficit. Otherwise, the excess power from the particular microgrid(s) is shared in proportion with the remaining microgrid(s). For example, consider the case for three microgrids which are interconnected, where microgrid 1 has an excess of $100 \mathrm{~W}$ but microgrids 2 and 3 have a power deficit of $120 \mathrm{~W}$ and $80 \mathrm{~W}$, respectively. In this case, the power deficit from microgrid 2 is $60 \%$ of the total deficit and hence it will receive $60 \mathrm{~W}$ from microgrid 1 whilst the remaining $40 \mathrm{~W}$ will be given to microgrid 3 .

Fig. 13 shows simulation results in this scenario, before and after the power sharing control is activated among the three microgrids which are linked with each other with a ring topology. It can be noticed that after enabling the power sharing scheme, the gap between the expected SoC (ideal case) and the real SoC becomes smaller. In order to quantify the impact, the resilience index was evaluated for three cases. In the first case, all the microgrids are interconnected i.e. power sharing among microgrids is possible. In the second case, microgrids are not interconnected i.e. each microgrid can only utilise its local resources. Finally, the resilience index was computed using the measurements and estimates of the real hardware.

Due to the implementation of the proposed dynamic penalty cost, load shedding is shifted towards the end of the scheduling horizon in all the cases. Therefore, resilience index values of only the last 12 intervals (10 minutes resolution) are shown in Table III, with the remaining intervals sharing the same values for all cases. Resilience index of each interval is computed for all the three cases using equations (11)-(13), which indicates the ability of the microgrid to survive loads during emergency operation.

According to (17), the acceptable resilience bound for all the microgrids of the network is $[0.63,1]$. This implies that all the critical loads need to survive during the emergency operation to ensure that the proposed resilience index is within the acceptable bounds. It can be observed from Table III that for the interconnected case, the acceptable bound is never violated throughout the islanded period due to ability of the microgrids to share the power. However, in the unconnected case, microgrid 1 and 3 are feeding all their loads (maximum resilience index value) while microgrid 2 is shedding even some of its critical loads in the last four intervals. Finally, in the hardware test case, the resilience index values of microgrid 1 and 3 are below the acceptable range due to deviation of the real $\mathrm{SoC}$ from the expected SoC of EMS. The resilience index of microgrid 2 is also below the acceptable range in the last interval. The SoC deviation in the experimental results was explained in the previous sections. As quantified in Table III, the SoC deviation resulted disconnection of critical loads.

\section{CONCLUSION}

This paper explores through simulation and experiment the limitations associated with energy management systems in microgrids. The objective was to study and demonstrate the fundamental discrepancies between real and estimated system states of an EMS which may compromise the resiliency of an interconnected microgrid system. First, time shifting, magnitude deviation and averaging effects were explored individually by calculating the power imbalance at each time step. Then, to mimic a more practical scenario, the elements were combined and as a result, a less predictable uncertainty profile was demonstrated. Using our laboratory-scaled microgrid system, an experimental test was conducted to demonstrate these issues in reality. The discrepancy between the measured battery SoC and the estimated SoC in the EMS was non-negligible and the difference becomes more pronounced for downstream microgrids due to the voltage 
drops in the interconnecting buses, which incurred higher losses. We quantified the impact of EMS uncertainties by using the proposed resiliency index parameter. The resiliency of the microgrid may be maintained, however, this may come at the expense of battery lifetime due to the higher DoD. Essentially, the trade-off is between additional cost incurred by shedding critical load and replacing batteries more often or installing higher battery capacity holds. This may require a long-term economic study to validate the hypothesis. Finally, the proposed power sharing scheme, demonstrated through simulation that it can mitigate the EMS uncertainties among the microgrids. Future work might consider more realistic extreme weather conditions, forecasting algorithms, and development of other control strategies that explicitly incorporate the issues discussed here.

\section{REFERENCES}

[1] J. Xiao, P. Wang, L. Setyawan, and Q. Xu, "Multi-Level Energy Management System for Real-Time Scheduling of DC Microgrids With Multiple Slack Terminals," IEEE Transactions on Energy Conversion, vol. 31, no. 1, pp. 392-400, Mar. 2016.

[2] Y. Zhang, R. Wang, T. Zhang, Y. Liu, and B. Guo, "Model predictive control-based operation management for a residential microgrid with considering forecast uncertainties and demand response strategies," IET Generation, Transmission \& Distribution, vol. 10, no. 10, pp. 23672378, Jul. 2016.

[3] T. Alnejaili, S. Drid, D. Mehdi, L. Chrifi-Alaoui, R. Belarbi, and A. Hamdouni, "Dynamic control and advanced load management of a standalone hybrid renewable power system for remote housing," Energy Conversion and Management, vol. 105, pp. 377-392, Nov. 2015.

[4] A. Tani, M. B. Camara, and B. Dakyo, "Energy Management in the Decentralized Generation Systems Based on Renewable EnergyUltracapacitors and Battery to Compensate the Wind/Load Power Fluctuations," IEEE Transactions on Industry Applications, vol. 51, no. 2, pp. 1817-1827, Mar.-Apr. 2015.

[5] J. Lee, P. Zhang, L. K. Gan, D. A. Howey, M. A. Osborne, A. Tosi, and S. Duncan, "Optimal operation of an energy management system using model predictive control and Gaussian process time-series modelling," IEEE Journal of Emerging and Selected Topics in Power Electronics, pp. 1-1, Mar. 2018.

[6] A. Parisio, C. Wiezorek, T. Kyntäjä, J. Elo, K. Strunz, and K. H. Johansson, "Cooperative MPC-Based Energy Management for Networked Microgrids," IEEE Transactions on Smart Grid, vol. 8, no. 6, pp. 3066-3074, Aug. 2017.

[7] S. R. Cominesi, M. Farina, L. Giulioni, B. Picasso, and R. Scattolini, "A Two-Layer Stochastic Model Predictive Control Scheme for Microgrids," IEEE Transactions on Control Systems Technology, vol. 26, no. 1, pp. 1-13, Jan. 2018.

[8] A. C. Luna, L. Meng, N. L. Diaz, M. Graells, J. C. Vasquez, and J. M. Guerrero, "Online Energy Management Systems for Microgrids: Experimental Validation and Assessment Framework," IEEE Transactions on Power Electronics, vol. 33, no. 3, pp. 2201-2215, Mar. 2018.

[9] D. Michaelson, H. Mahmood, and J. Jiang, "A Predictive Energy Management System Using Pre-Emptive Load Shedding for Islanded Photovoltaic Microgrids," IEEE Transactions on Industrial Electronics, vol. 64, no. 7, pp. 5440-5448, Mar. 2017.

[10] B. Zhao, X. Wang, D. Lin, M. Calvin, J. Morgan, R. Qin, and C. Wang, "Energy Management of Multiple-Microgrids based on a System of Systems Architecture," IEEE Transactions on Power Systems, pp. 1-1, May. 2018.

[11] L. K. Gan, J. K. H. Shek, and M. A. Mueller, "Optimised operation of an off-grid hybrid wind-diesel-battery system using genetic algorithm," Energy Conversion and Management, vol. 126, pp. 446-462, Oct. 2016.

[12] B. V. Solanki, K. Bhattacharya, and C. A. Cañizares, "A Sustainable Energy Management System for Isolated Microgrids," IEEE Transactions on Sustainable Energy, vol. 8, no. 4, pp. 1507-1517, Oct. 2017.

[13] A. Gholami, T. Shekari, and S. Grijalva, "Proactive Management of Microgrids for Resiliency Enhancement: An Adaptive Robust
Approach," IEEE Transactions on Sustainable Energy, pp. 1-1, Aug. 2017.

[14] Z. Wang and J. Wang, "Self-Healing Resilient Distribution Systems Based on Sectionalization Into Microgrids," IEEE Transactions on Power Systems, vol. 30, no. 6, pp. 3139-3149, Nov. 2015.

[15] A. Hussain, V. H. Bui, and H. M. Kim, "Optimal operation of hybrid microgrids for enhancing resiliency considering feasible islanding and survivability," IET Renewable Power Generation, vol. 11, no. 6, pp. 846857, May 2017.

[16] M. Panteli, D. N. Trakas, P. Mancarella, and N. D. Hatziargyriou, "Power Systems Resilience Assessment: Hardening and Smart Operational Enhancement Strategies," Proceedings of the IEEE, vol. 105, no. 7, pp. 1202-1213, Jul. 2017.

[17] L. Kleerekoper, M. Taleghani, A. van den Dobbelsteen, and T. Hordijk, "Urban measures for hot weather conditions in a temperate climate condition: A review study," Renewable and Sustainable Energy Reviews, vol. 75, pp. 515-533, Aug. 2017.

[18] (2017). "United States' electric grid remains vulnerable to natural disasters, cyber and physical attacks; actions needed to improve resiliency of the power system, Washington, DC: National Academy of Sciences," Available: http://nap.edu/24836

[19] S. Dunn, S. Wilkinson, D. Alderson, H. Fowler, and C. Galasso, "Fragility Curves for Assessing the Resilience of Electricity Networks Constructed from an Extensive Fault Database," Natural Hazards Review, vol. 19, no. 1, pp. 1 - 10, Feb. 2018.

[20] M. Panteli, C. Pickering, S. Wilkinson, R. Dawson, and P. Mancarella, "Power System Resilience to Extreme Weather: Fragility Modeling, Probabilistic Impact Assessment, and Adaptation Measures," IEEE Transactions on Power Systems, vol. 32, no. 5, pp. 3747-3757, Sept. 2017.

[21] P. Venkataraman, Applied Optimization with MATLAB Programming, 2nd ed.: John Wiley \& Sons, 2009.

[22] D. A. Coley, An Introduction to Genetic Algorithms for Scientists and Engineers: World Scientific, 1999.

[23] W. Al-Saedi, S. W. Lachowicz, D. Habibi, and O. Bass, "Power flow control in grid-connected microgrid operation using Particle Swarm Optimization under variable load conditions," International Journal of Electrical Power \& Energy Systems, vol. 49, pp. 76-85, Jul. 2013.

[24] J. Arora, Introduction to Optimum Design, 3rd ed.: Academic Press, 2011.

[25] S. M. Dawoud, X. Lin, and M. I. Okba, "Hybrid renewable microgrid optimization techniques: A review," Renewable and Sustainable Energy Reviews, vol. 82, pp. 2039-2052, Feb. 2018.

[26] E. McKenna and M. Thomson, "High-resolution stochastic integrated thermal-electrical domestic demand model," Applied Energy, vol. 165, pp. 445-461, Mar. 2016.

[27] I. Richardson, M. Thomson, D. Infield, and C. Clifford, "Domestic electricity use: A high-resolution energy demand model," Energy and Buildings, vol. 42, no. 10, pp. 1878-1887, Oct. 2010.

[28] L. K. Gan, B. Riar, J. Lee, and D. Howey, "Low-cost modular PV-battery microgrid emulator for testing of energy management algorithms," in 2017 IEEE Second International Conference on DC Microgrids (ICDCM), Jun. 2017, pp. 602-608.

[29] D. J. Spiers and A. D. Rasinkoski, "Predicting the service lifetime of lead/acid batteries in photovoltaic systems," Journal of Power Sources, vol. 53, no. 2, pp. 245-253, Feb. 1995.

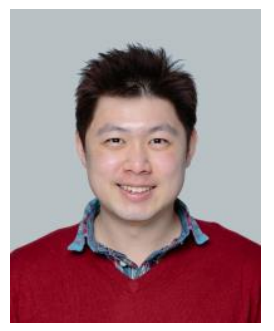

Leong Kit Gan (M'17) received the B.Eng. degree (Hons.) in electrical and electronics engineering from the Universiti Tenaga Nasional, Kuala Lumpur, Malaysia, in 2009, the M.Sc. degree in sustainable energy systems and the Ph.D. degree in energy systems from The University of Edinburgh, Edinburgh, U.K., in 2012 and 2016, respectively.

From 2016 to 2018, he was a Postdoctoral Researcher at the University of Oxford, focusing on intelligent control of decentralised energy systems. Between 2009 and 2011, he was an Electronics Engineer in Altera and Motorola.

His research interests include control of power electronics, energy storage and renewable energy systems. 


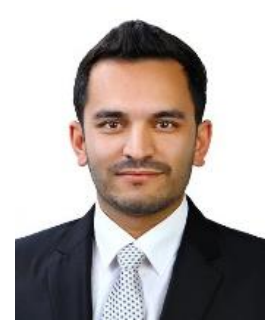

Akhtar Hussain (S'14) received his B.E degree in Telecommunications from the National University of Sciences and Technology (NUST) Pakistan in 2011 and his M.S in Electrical Engineering from Myongji University, Yongin, South Korea, in 2014. Currently, he is a Ph.D. student at the Incheon National University, Korea. His research interests are power system automation, smart grids, and microgrid operation.

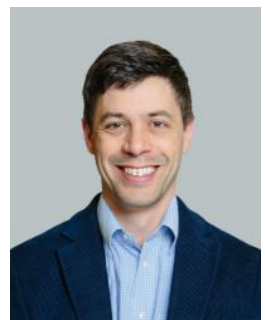

David A. Howey (M'10) received the B.A. and M.Eng. degrees from Cambridge University, Cambridge, U.K., in 2002 and the Ph.D. degree from Imperial College London, London, U.K., in 2010. He is currently an Associate Professor with the Energy and Power Group, Department of Engineering Science, University of Oxford, Oxford, U.K. His research is focused primarily on energy storage systems, including projects on model-based battery management, degradation, thermal management, and energy management for grid storage.

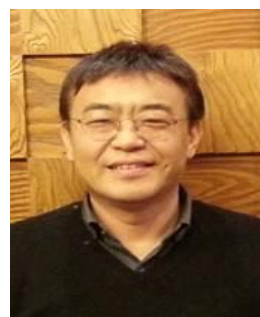

Hak-Man Kim (SM'15) received his first Ph.D. degree in Electrical Engineering from Sungkyunkwan University, Korea in 1998 and received his second $\mathrm{Ph} . \mathrm{D}$. degree in Information Sciences from Tohoku University, Japan, in 2011, respectively. He worked for Korea Electrotechnology Research Institute (KERI), Korea from Oct. 1996 to Feb. 2008. Currently, he is a professor in the Department of Electrical Engineering, Incheon National University, Korea. His research interests include microgrid operation \& control and DC power systems. 